\title{
Processamento sensorial e oportunidades para o desenvolvimento de bebês
}

\section{Sensory processing and environmental oppotunities for babies development}

\author{
Luana Borges Britto ${ }^{1}$, Camila Boarini dos Santos ${ }^{2}$, Danielle dos Santos Cutrim Garros ${ }^{3}$, \\ Aila Narene Dahwache Criado Rocha ${ }^{4}$
}

http://dx.doi.org/10.11606/issn.2238-6149.v31i1-3p9-16

Britto LB, Santos CB, Garros DSC, Rocha ANDC. Práticas e desafios para a terapia ocupacional no contexto da Intervenção Precoce Processamento sensorial e oportunidades para o desenvolvimento de bebês. Rev Ter Ocup Univ São Paulo. 2020 jan-dez;31(1-3):9-16.

RESUMO: O objetivo foi caracterizar o processamento sensorial e as oportunidades ambientais de bebês. A pesquisa foi submetida à avaliação para o Comitê de Ética, tendo parecer favorável. A coleta de dados foi realizada durante visitas domiciliares e participaram da pesquisa 21 bebês e seus responsáveis. Foi utilizado o Perfil Sensorial 2 do Bebê e da Criança Pequena para avaliar o processamento sensorial e o Affordances in the home environment for motor development para avaliar as oportunidades ambientais. Após a análise dos instrumentos, os dados foram compilados no programa Microsoft $\mathrm{Excel}^{\circ}$ e posteriormente foi realizada a análise descritiva. Os resultados evidenciaram que, mesmo avaliando bebês sem diagnóstico que interferisse no desenvolvimento infantil, em alguns casos, apresentaram alterações no processamento sensorial. Em relação às oportunidades ambientais, foram encontradas residências com a classificação "fraca" e "muito fraca". Foi concluído que há a necessidade do monitoramento do desenvolvimento de bebês pela Atenção Primária em Saúde, mesmo sem o indicativo de atraso no desenvolvimento.

DESCRITORES: Desenvolvimento infantil; Transtornos das sensações; Atenção primária à saúde; Terapia ocupacional.
Britto LB, Santos CB, Garros DSC, Rocha ANDC. Sensory processing and environmental oppotunities for babies development. Rev Ter Ocup Univ São Paulo. 2020 Jan-Dec;31(1-3):9-16.

\begin{abstract}
The aim of the study was to characterize the sensory processing and environmental opportunities of babies. The research was submitted to evaluation by the Ethics Committee, having a favorable opinion. Data collection was performed during home visits and 21 babies and their guardians participated in the research. The Baby and Toddler Sensory Profile 2 was used to assess sensory processing and Affordances in the home environment for motor development to assess environmental opportunities. After the analysis of the instruments, the data were compiled in the Microsoft Excel $^{\circ}$ program and later a descriptive analysis was performed. The results showed that, even evaluating babies without a diagnosis that interfered with child development, in some cases, presented altered sensory processing. Regarding environmental opportunities, residences with the classification "weak" and "very weak" were found. It was concluded that there is a need for monitoring on the development of babies by Primary Health Care, even without the indication of developmental delay.
\end{abstract}

KEYWORDS: Child development; Sensation disorders; Primary health care; Occupational therapy.

Apresentado no X Encontro Científico de Terapia Ocupacional, UNESP, Marília, 28-29 nov. 2019.

1. Terapeuta ocupacional. Graduada pela Universidade Estadual Paulista Júlio de Mesquita Filho, UNESP, São Paulo, Brasil. Orcid: https://orcid.org/0000-0002-9501-4872. E-mail: lua_na-brito@hotmail.com.

2. Terapeuta Ocupacional. Mestra em Educação pelo Programa de Pós Graduação em Educação e colaboradora do Laboratório de Estudo em Acessibilidade, Tecnologia Assistiva e Inclusão (LATAI) pela Universidade Estadual Paulista Júlio de Mesquita Filho. São Paulo, Brasil. Orcid: https://orcid.org/0000-0001-5594-0305. E-mail: camila.boarini@unesp.br.

3. Docente e Pesquisadora do Departamento de Fisioterapia e Terapia Ocupacional (DEFITO), Universidade Estadual Paulista Júlio de Mesquita Filho, UNESP. São Paulo, Brasil. Orcid: https://orcid.org/0000-0003-1525-0056. E-mail: danielle.garros@unesp.br.

4. Docente e Pesquisadora do Departamento de Fisioterapia e Terapia Ocupacional (DEFITO), coordenadora do Laboratório de Estudo em Acessibilidade, Tecnologia Assistiva e Inclusão (LATAI)pela Universidade Estadual Paulista Júlio de Mesquita Filho. São Paulo, Brasil. Orcid: https://orcid.org/0000-0001-6186-875X. E-mail: aila.rocha@unesp.br.

Endereço para correspondência: Rua Angelina Dal Lago, nº 206 Bairro: Parque das Esmeralda II, Marília, São Paulo. CEP: 17516-730. 


\section{INTRODUÇÃO}

$\mathrm{P}$ ara desenvolver habilidades motoras, afetivas e cognitivas, o Sistema Nervoso Central (SNC) deve interagir em um processo contínuo de feedback às respostas dos órgãos sensitivos. A ocorrência de déficits na recepção dos estímulos sensoriais, por um mal funcionamento fisiológico ou anatômico dos órgãos sensitivos, ou ao nível do SNC, por déficits de processamento sensorial, poderá interferir no desenvolvimento neuropsicomotor (DNPM), em especial nas habilidades motoras e cognitivas ${ }^{1,2}$. O processamento sensorial é responsável pela recepção dos estímulos, modulação, integração, discriminação e organização das sensações, possibilitando uma resposta adaptativa às situações experimentadas ${ }^{3}$.

$\mathrm{O}$ equilíbrio entre limiares de respostas de um estímulo é determinante no processo de aprendizagem. A recepção de estímulos e respostas do processamento sensorial permite que as pessoas percebam situações a sua volta, de forma que não fiquem sobrecarregas com tais informações ou distraídas. Quando o bebê responde rapidamente a um estímulo sensorial é entendido que há um limiar baixo e quando essa resposta é mais lenta do que o esperado, que há um limiar elevado ${ }^{4}$.

A habituação, sensibilização e a capacidade de modular as respostas do processamento sensorial são significativas para o DNPM do bebê. A habituação, principalmente do bebê, permite que ele foque sua atenção à uma atividade específica, para que esta possa ser aperfeiçoada até sua realização de forma automática. A sensibilização é o processo que aumenta a consciência de estímulos importantes e permite que o bebê permaneça atento ao ambiente enquanto está focado em uma tarefa. A organização e o equilíbrio das informações recebidas por meio dos estímulos são chamados de modulação. A capacidade de modular as respostas do processamento sensorial permite que a criança gere uma resposta adequada para cada estímulos no ambiente ${ }^{4}$.

O processamento sensorial é considerado adequado quando todos os receptores sensoriais interagem adequadamente, o que pode ser observado por meio dos comportamentos. Para a organização do comportamento é necessária uma sequência de ações em relação ao espaço e tempo, para tal organização utilizamos a práxis. A práxis é a capacidade de gerar, organizar e realizar ações, ou seja, a produção de atividades como base nas informações derivadas do processamento sensorial, obtidas através dos órgãos sensitivos e do ambiente ${ }^{2,5}$.

A percepção depende das informações fornecidas pelo ambiente, para que o bebê a receba e processe. Além do processamento sensorial é importante ressaltar que um ambiente que oferte estímulos de forma equilibrada, favorecerá o desenvolvimento de comportamentos adequados do bebê ${ }^{2}$.

$\mathrm{O}$ artigo de Caçola et al. ${ }^{6}$ teve o objetivo de apresentar $\mathrm{o}$ instrumento Affordances in the home environment for motor development/ (Oportunidades de estimulação motora na casa familiar (AHEMD) que, a partir do relato dos pais, avalia as oportunidades ambientais presentes nas residências familiares, de forma quantitativa e qualitativa, que possam interferir no desenvolvimento do bebê.

Os estudos de Almeida et al. ${ }^{7}$, Caçola et al. ${ }^{8}$, Pedrosa et al. ${ }^{5} \mathrm{e}$ Pereira ${ }^{9}$ investigaram como as oportunidades ambientais interferem em algum aspecto do desenvolvimento infantil. Ambos sugeriram a ampliação de estudos na área, pois as oportunidades ambientais não têm sido avaliadas de forma satisfatória. Em relação ao desenvolvimento infantil, os estudos demonstraram que as oportunidades ambientais estão abaixo do que é necessário para a promoção do desenvolvimento do bebêt $\hat{e}^{7,8,5,9}$.

O estudo de Molini-Avejonas et al. ${ }^{10}$ demonstra que existem lacunas no monitoramento do desenvolvimento de bebês na Atenção Primária, visto que, identificar e avaliar fatores de risco para o desenvolvimento do bebê e orientar sobre a relação do ambiente como forma de estimular o desenvolvimento, são maneiras eficazes de prevenir e intervir precocemente em possíveis atrasos do desenvolvimento infantil.

O ápice do desenvolvimento ocorre de 0 a 24 meses e é nessa fase que o bebê tem suas habilidades cognitivas e motoras em evolução. Tal informação é base para justificar a importância do acompanhamento do desenvolvimento do bebê, visando à prevenção de possíveis atrasos do desenvolvimento e planejar intervenções precoces, caso necessário ${ }^{11}$.

Justifica-se a relevância deste estudo pela necessidade de ampliar as evidências cientificas nessa área, visto que, o monitoramento do desenvolvimento do bebê, bem como avaliar o processamento sensorial, tem a proposta de orientar os cuidadores sobre maneiras de estimular, prevenir e identificar possíveis atrasos no desenvolvimento infantil e investigar os níveis de estímulos presentes no ambiente em que o bebê vive ${ }^{7,8,5,9}$.

Desta forma, pretendeu-se investigar qual o perfil de desenvolvimento do processamento sensorial e das oportunidades ambientais presentes nas residências de bebês. Acredita-se, como hipótese, que por se tratar de bebês atermos sem nenhum diagnóstico que justifique possíveis atrasos no desenvolvimento do processamento sensorial e as oportunidades ambientais oferecidas, os bebês estarão dentro do esperado de acordo com a sua idade cronológica.

Portanto, este estudo teve como objetivo caracterizar o processamento sensorial e as oportunidades ambientais de bebês. 


\section{Procedimentos Metodológicos}

O projeto de pesquisa foi submetido à avaliação pelo Comitê de Ética em Pesquisa da Faculdade de Filosofia e Ciências da UNESP - Campus de Marília/SP, respeitando as prerrogativas da resolução 510/16 do CONEP que versa sobre ética em pesquisa com seres humanos tendo parecer favorável sob o CAAE: 09740319.4.0000.5406.

Foi encaminhado um ofício ao Centro Especializado de Reabilitação vinculado a Universidade Estadual Paulista (UNESP), na cidade de Marília, solicitando permissão para realização da pesquisa. Após autorização, foi identificado a lista de bebês acompanhados pelo Programa de Aprimoramento Profissional desta instituição.

A fim de prestar esclarecimentos sobre os objetivos, procedimentos de coleta de dados, tempo de duração, resguardo da privacidade dos participantes e utilização dos dados para fins científicos, os responsáveis foram convidados a assinar o termo de consentimento livre e esclarecido.

A coleta de dados foi realizada através de visitas domiciliares e participaram da pesquisa os pais de 21 bebês de 0 a 24 meses, no período de abril a junho de 2019.

O critério de inclusão dos participantes deste estudo foi que os bebês avaliados pertencessem à faixa etária do estudo. Essas informações foram obtidas por meio da análise de prontuários e histórico referido pela equipe de profissionais responsáveis pelos casos. Os critérios de exclusão foram os bebês que tinham deficiências, possíveis atrasos no desenvolvimento e/ou nascidos pré-termo.

Para avaliar o processamento sensorial utilizou-se dois instrumentos de avaliação: A) Perfil Sensorial 2 do Bebê: possui 25 itens sobre o bebê, desde o nascimento até seis meses de idade; B) Perfil Sensorial 2 da Criança Pequena: possui 54 itens sobre a criança pequena, desde sete até 35 meses de idade. Esses instrumentos de avaliação são padronizados para que profissionais documentem, de acordo com a visão do cuidador, os padrões de processamento sensorial do bebê. Estes dados apoiam a equipe de profissionais a acompanhar o desenvolvimento do processamento sensorial do bebê, considerando sua participação nas atividades ${ }^{4}$.

Os instrumentos analisam a frequência de comportamentos do bebê durante as atividades que envolvem experiências sensoriais nos diferentes contextos. Para análise, foi utilizada a escala Likert para indicar a opção que melhor descreve a frequência na qual o bebê demonstra determinado comportamento, tendo como respostas as seguintes alternativas: 1$)$ "Quase nunca" ( $10 \%$ ou menos do tempo); 2) "Ocasionalmente" (25\% do tempo); 3 ) "Metade do tempo" ( $50 \%$ do tempo); 4) "Frequentemente" ( $75 \%$ do tempo); e 5) "Quase sempre" ( $90 \%$ ou mais do tempo) $)^{4}$.
O perfil Sensorial 2 do Bebê fornece uma pontuação total de acordo com os sistemas sensoriais (Processamento geral; Processamento auditivo; Processamento visual; Processamento do tato; Processamento de movimentos; e Processamento da sensibilidade oral). O Perfil Sensorial 2 da Criança Pequena avalia os mesmos sistemas sensoriais contidos no Perfil Sensorial 2 do Bebê, além do processamento comportamental e os quatro quadrantes (Exploração; Esquiva; Sensibilidade; e Observação) que classificam o grau em que os bebês respondem aos estímulos sensoriais. Em relação aos quadrantes, a autora do instrumento define que: o quadrante Exploração analisa o grau em que a criança obtém estímulo sensorial; o quadrante Esquiva avalia o quanto a criança se incomoda com os estímulos sensoriais; o quadrante Sensibilidade avalia o grau em que a criança detecta um estímulo sensorial e; o quadrante Observação identifica o grau em que a criança deixa de perceber os estímulos sensoriais ${ }^{4}$.

Como instrumento para avaliar as oportunidades que a residência familiar oferece para o desenvolvimento do bebê foi utilizado a AHEMD, que é considerada uma ferramenta confiável e padronizada que permite avaliar de forma quantitativa e qualitativa as oportunidades de estimulação motora no ambiente domiciliar ${ }^{6}$.

Foram utilizadas as duas versões do AHEMD: a escala bebê, que corresponde de 3 a 18 meses e a escala de 18 a 42 meses, contemplando a idade avaliada na pesquisa. Os questionários foram respondidos pelos cuidadores. A AHEMD é dividida em cinco categorias, sendo elas: características do bebê e da família; Características e dimensões do espaço físico externo e interno da residência familiar; Frequência de atividades diárias da família com o bebê; e Materiais e brinquedos que estimulam a motricidade fina e grossa presentes nas residências.

Foi utilizado, como forma de resposta, os tipos dicotômicas simples (sim/não), escala Likert (com quatro níveis de respostas como: "quase sempre", "muito tempo", "pouco tempo"e "quase nunca" - como objetivo de descrever a quantidade de tempo em que a bebê passa acordado em diferentes situações diárias) e questões descritivas com ilustrações com modelos de diversos tipos de brinquedos e materiais utilizados e presentes nas residências dos bebês ${ }^{5}$.

$\mathrm{Na}$ escala de 3 a 18 meses o instrumento permite dois tipos de respostas, a escolha dicotômica (sim/não) nas dimensões "espaço externo", "espaço interno" e em duas questões da dimensão "variedade de estimulação". Ainda na dimensão "variedade de estimulação" é utilizada nas demais questões a quantificação do tempo em que a criança passa acordada em determinada situação (nunca; às vezes; quase sempre e sempre), e nas dimensões "brinquedos para 
motricidade grossa" e "brinquedos para motricidade fina" a quantificação dos brinquedos que estão presentes nas residências (nenhum; um a dois; e três ou mais).

A classificação total das oportunidades ambientais é derivada da soma da pontuação total de cada dimensão. $\mathrm{Na}$ avaliação 3 a 18 meses a pontuação final classifica as oportunidades da residência como "menos que adequado", "moderadamente adequado", "adequado" e "excelente". Na avaliação de 18 a 42 meses a pontuação final permite classificar das oportunidades das residências como, "Muito fraca", "fraca", "média", "boa" e "muito boa". Neste estudo, para melhor visualização dos resultados, foi utilizado uma única classificação para os dois instrumentos, sendo utilizado a proposta da avaliação de 18 a 42 meses, adequando da seguinte forma: "menos que adequado" classificada como "fraca"; "moderadamente adequado" classificada como "média"; "adequada" classificada como "boa"; e "excelente" classificada como "muito boa".

Para a coleta de dados iniciou-se a aplicação dos questionários do Perfil Sensorial 2, com os responsáveis do bebê. A primeira visita domiciliar teve duração de aproximadamente 1 hora e 30 minutos. Ao final foi agendado a segunda vista domiciliar para a realização da avaliação AHEMD com, aproximadamente, 40 minutos de duração, sendo também e esclarecidas eventuais dúvidas a respeito do estudo. Todas as avaliações foram realizadas por meio de entrevistas com os responsáveis pelos bebês para preenchimento dos questionários padronizados elou pela observação das atividades dos bebês, quando necessário.

Para análise de dados foram respeitadas a pontuação e classificação propostas por cada instrumento. Após a análise dos instrumentos, os dados foram compilados no programa Microsoft Excel ${ }^{\mathcal{O}}$ e posteriormente foi realizada a análise estatística descritiva, com objetivo de caracterizar o processamento sensorial e as oportunidades ambientais oferecidas ao bebê. Para análise descritiva do instrumento Perfil Sensorial 2 foi proposto a junção das categorias "muito menos do que outros" e "menos do que outros" e a junção das categorias "mais do que outros" e "muito mais do que outros".

Os resultados das oportunidades ambientais estão classificados de 1 a 5 , sendo 1 "muito fraca"; 2 "fraca"; 3 "média"; 4 "boa"; e 5 "muito boa" às oportunidades ambientais que favorecem o desenvolvimento do bebê.

\section{RESULTADOS}

Os resultados deste estudo serão apresentados pela caracterização dos participantes (Quadro 1); Perfil Sensorial e Oportunidades Ambientais.

Dentre os 21 bebês (Quadro 1), quatro não foram avaliados nas categoriais dos quadrantes e no processamento sensorial comportamental, pois com esses participantes foi aplicado o Perfil Sensorial 2 do Bebê, ou seja, tais categorias não são avaliadas no instrumento adequado à esta faixa etária dos bebês. Ressalta-se que as classificações que revelam comportamentos menos frequentes que o esperado para a idade e mais frequentes que o esperado para a idade necessita de atenção $0^{4}$.

Quadro 1 - Caracterização dos bebês

\begin{tabular}{|c|c|c|c|c|}
\hline Bebês & Idade & Sexo & Renda Familiar & $\begin{array}{c}\text { Frequenta } \\
\text { a creche }\end{array}$ \\
\hline B1 & 3 meses & $\mathrm{F}$ & $\mathrm{R} \$ 2.500$ a $\mathrm{R} \$ 3.500$ & Não \\
\hline $\mathrm{B} 2$ & 5 meses & $\mathrm{F}$ & $\mathrm{R} \$ 1.000 \mathrm{a} \mathrm{R} \$ 1.500$ & Não \\
\hline B3 & 5 meses & M & $\mathrm{R} \$ 1.500$ a $\mathrm{R} \$ 2.500$ & Não \\
\hline B4 & 6 meses & $\mathrm{F}$ & $\mathrm{R} \$ 2.500$ a $\mathrm{R} \$ 3.500$ & Não \\
\hline B5 & 7 meses & M & $\mathrm{R} \$ 2.500$ a $\mathrm{R} \$ 3.500$ & Não \\
\hline B6 & 10 meses & $\mathrm{F}$ & $\mathrm{R} \$ 2.500$ a $\mathrm{R} \$ 3.500$ & Não \\
\hline B7 & 10 meses & M & $\mathrm{R} \$ 1.500$ a $\mathrm{R} \$ 2.500$ & Não \\
\hline B8 & 13 meses & M & $\mathrm{R} \$ 2.500 \mathrm{a} R \$ 3.500$ & Não \\
\hline B9 & 13 meses & M & $\mathrm{R} \$ 1.000$ a $\mathrm{R} \$ 1.500$ & Não \\
\hline $\mathrm{B} 10$ & 17 meses & $\mathrm{F}$ & $\mathrm{R} \$ 2.500$ a $\mathrm{R} \$ 3.500$ & Não \\
\hline B11 & 16 meses & $\mathrm{F}$ & $\mathrm{R} \$ 1.000 \mathrm{a} \mathrm{R} \$ 1.500$ & Não \\
\hline B12 & 16 meses & M & $\mathrm{R} \$ 1.000$ a $\mathrm{R} \$ 1.500$ & Não \\
\hline B13 & 17 meses & M & $\mathrm{R} \$ 1.000$ a $\mathrm{R} \$ 1.500$ & Não \\
\hline B14 & 18 meses & M & $\mathrm{R} \$ 1.500$ a $\mathrm{R} \$ 2.500$ & Não \\
\hline B15 & 20 meses & $\mathrm{F}$ & $\mathrm{R} \$ 1.500$ a $\mathrm{R} \$ 2.500$ & Sim \\
\hline B16 & 21 meses & M & $\mathrm{R} \$ 1.000$ a $\mathrm{R} \$ 1.500$ & Sim \\
\hline B17 & 21 meses & $\mathrm{F}$ & $\mathrm{R} \$ 2.500$ a $\mathrm{R} \$ 3.500$ & Sim \\
\hline B18 & 22 meses & $\mathrm{F}$ & $\mathrm{R} \$ 1.500$ a $\mathrm{R} \$ 2.500$ & Não \\
\hline B19 & 22 meses & $\mathrm{F}$ & $\mathrm{R} \$ 2.500$ a $\mathrm{R} \$ 3.500$ & Sim \\
\hline $\mathrm{B} 20$ & 23 meses & M & $\mathrm{R} \$ 1.500$ a $\mathrm{R} \$ 2.500$ & Sim \\
\hline B21 & 23 meses & $\mathrm{F}$ & $\mathrm{R} \$ 2.500$ a $\mathrm{R} \$ 3.500$ & Sim \\
\hline
\end{tabular}

Fonte: Elaboração própria.

O percentual de cada categoria do processamento sensorial está descrito na Figura 1 (Processamento Geral, Auditivo, Oral, Visual, Tátil, Movimentos, Comportamental), no qual mostrou que, no "processamento geral", $80 \%$ dos bebês pontuaram no Perfil Sensorial como "Exatamente como a maioria dos outros", $10 \%$ pontuaram "Muito menos do que outros/menos do que outros" e 10\% "Mais do que outros/ muito mais do que outros". No "processamento auditivo" e "processamento oral" $95 \%$ dos bebês apresentaram o resultado "Exatamente como a maioria dos outros" e 5\% dos bebês apresentaram um limiar abaixo do esperado para a faixa etária. 

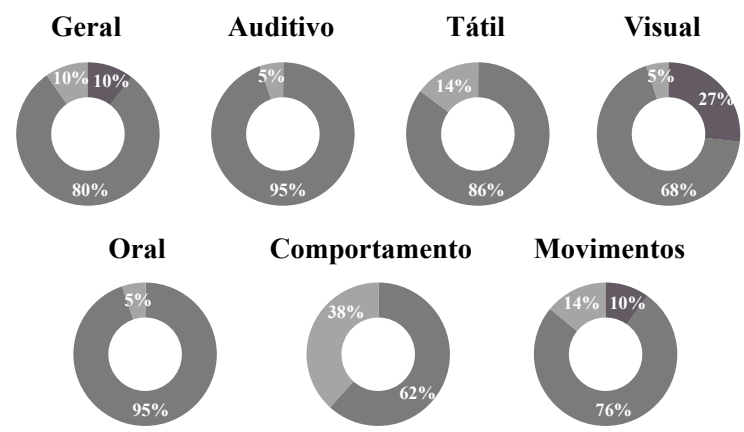

Comportamento

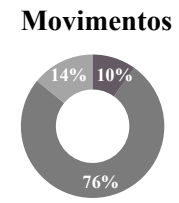

Muito menos do que outros/Menos do que outros Exatamente como a maioria dos outros Mais do que outros/Muito mais do que outros

Fonte: Elaboração própria.

Figura 1 - Resultados das áreas de Processamento Sensorial

No "processamento visual" $68 \%$ dos bebês revelaram estar com o processamento sensorial dentro do limiar adequado, "Exatamente como a maioria dos outros", $27 \%$ dos bebês apresentaram resultado "Muito menos do que outros/menos do que outros" e $5 \%$ foram classificados como "Mais do que outros/muito mais do que outros".

O resultado do "processamento tátil" relevou que $86 \%$ dos bebês apresentaram um limiar "Exatamente como a maioria dos outros" e $14 \%$ um limiar acima do esperado "Mais do que outros/muito mais do que outros". No "processamento de movimentos (vestibular)" 76\% apresentaram resultado "Exatamente como a maioria dos outros", $14 \%$ pontuaram "Mais do que outros/muito mais do que outros" e 10\% "Muito menos do que outros/menos do que outros". A categoria com o maior percentil do resultado "Mais do que outros/ Muito mais do que outros" foi a comportamental, com $38 \%$ dos bebês, $62 \%$ apontou estar "Exatamente como a maioria dos outros".

Os resultados percentuais dos quadrantes do Perfil Sensorial 2 estão descritos na Figura 2 (Observação, Sensibilidade, Esquiva, Exploração), demonstrando que, no quesito "Observação", $100 \%$ dos bebês, apresentaram estar "Exatamente como a maioria dos outros".

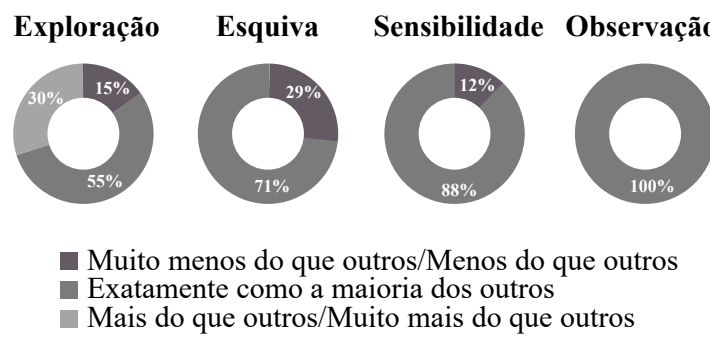

Fonte: Elaboração própria.

Figura 2 - Resultados dos Quadrantes do Processamento Sensorial
Já no quadrante "Sensibilidade" $88 \%$ dos bebês revelaram estar "Exatamente como a maioria dos outros" e $12 \%$ se enquadraram em "Muito menos do que outros/ Menos do que outros". No quadrante "Esquiva" 71\% obtiveram o resultado "Exatamente como a maioria dos outros" e 29\% "Muito menos do que outros/ Menos do que outros". No quadrante "Exploração" 55\% dos bebês apresentaram estar no limiar dentro do esperado para idade, "Exatamente como a maioria dos outros", 30\% apresentaram um limiar alto "Mais do que outros/ Muito mais do que outros e $15 \%$ revelaram estar com um limiar baixo, "Muito menos do que outros/ Menos do que outros.

A avaliação das oportunidades ambientais demonstrou que $67 \%$ das residências familiares obtiveram a classificação "média" das oportunidades ambientais, $19 \%$ foram classificadas como "boa" e $14 \%$ classificadas como "fraca" as oportunidades que favorecem o desenvolvimento do bebê, como descrito na Figura 3.

\section{Classificação Geral}

1 Muito Fraca $\square 2$ Fraca $\square 3$ media $\square 4$ Boa $\square 5$ Muito Boa

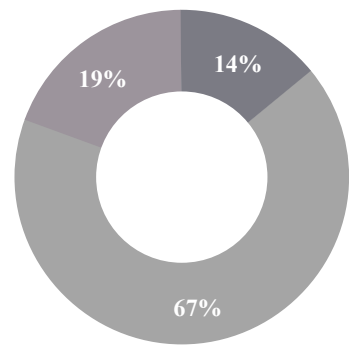

Fonte: Elaboração própria.

Figura 3 - Classificação geral das oportunidades ambientais

A Figura 4 demonstra o percentual das classificações de cada categoria avaliada na AHEMD.

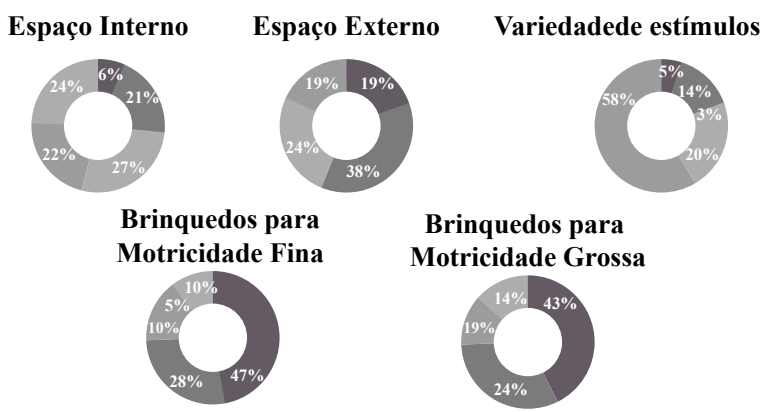

1 Muito Fraca $\square 2$ Fraca $\square 3$ Média $\square 4$ Boa $\square 5$ Muito Boa Fonte: Elaboração própria.

Figura 4 - Resultados das categorias da AHEMD 
O "Espaço interno" teve como resultado que 27\% das residências foram classificadas como "média", $21 \%$ como "fraca" e $6 \%$ como "muito fraca". Já na categoria "Espaço externo" 38\% das residências foram classificadas como "fraca" e $24 \%$ como "média".

Na categoria "Variedades de Estímulos" 5\% das residências foram classificadas como "muito fraca", 14\% classificadas como "fraca" e 3\% das residências foram classificadas como "média".

Dentre as categorias das oportunidades ambientais que tiveram o maior percentual negativo foram os "Brinquedos para Motricidade Fina" e "Brinquedos para Motricidade Grossa". Na categoria "Brinquedos para Motricidade Fina" $47 \%$ das residências foram classificadas como "muito fraca" as oportunidades para motricidade fina e $28 \%$ como "fraca" e $10 \%$ como "média". Na categoria "Brinquedos para Motricidade Grossa" $43 \%$ das residências foram classificadas como "muito fraca", $24 \%$ classificadas como "fraca" e 19\% como "média".

\section{DISCUSSÃO}

Os resultados evidenciaram que, mesmo avaliando bebês que não possuem nenhum diagnóstico médico que pudesse interferir no desenvolvimento infantil, obteve-se resultados não esperados para o público analisado. Portanto, detectar precocemente disfunções no processamento sensorial nos permite fornecer intervenções apropriadas que poderão favorecer o desenvolvimento infantil. O sistema sensorial tem relação direta com a participação e desempenho do bebê nas suas atividades diárias e ocupações e a Terapia Ocupacional tem um papel fundamental no monitoramento do processamento sensorial do bebê, visto que são profissionais habilitados para compreender e avaliar a relação do sistema sensorial com a funcionalidade de bebê. $\hat{e}^{12,13}$.

$\mathrm{O}$ estudo de Pedrosa et al..$^{5}$ identificou os fatores ambientais preditores do Perfil Sensorial de lactentes dos quatro aos 18 meses de idade. De 97 participantes, 66\% apresentaram processamento sensorial normal e 34\% revelaram riscos ou déficits no processamento sensorial. Em comparação com o presente estudo, os resultados foram semelhantes e indicam a necessidade de acompanhamento e encaminhamento para profissionais específicos e reavaliações para o monitoramento do processamento sensorial ${ }^{6}$.

Cabral et al. ${ }^{14}$ avaliou e comparou o processamento sensorial de 30 crianças, sendo 15 nascidas pré-termo e 15 nascidas termo, utilizando como instrumento de avaliação o Test of Senrory Function in Infants (TSFI). O grupo de nascidos termo revelou, em alguns casos, a classificação "deficiente" ou de "risco" sobre o processamento sensorial, resultados não esperados para bebês com desenvolvimento típico.
Com o objetivo de analisar a correlação entre Perfil Sensorial do bebê, idade gestacional e risco psíquico e seus efeitos na avaliação motora e de linguagem, Beltrame et al. ${ }^{15}$ avaliou 40 bebês, 20 nascidos pré-termo e 20 nascidos termo. $\mathrm{O}$ grupo controle de nascidos termo obtiveram como resultados um percentual de bebês com alteração no processamento sensorial.

Todas as áreas do processamento sensorial deste estudo, apontaram uma porcentagem dos bebês com o desenvolvimento não esperado para idade, com alterações para mais e/ou para menos do limiar adequado.

A literatura aponta que tanto os bebês prematuros, quanto os bebês com desenvolvimento típico apresentaram alterações em alguma área do processamento sensorial, porém existe uma tendência maior para os bebês nascidos pré-termo. Desta forma, fica evidente que bebês com desenvolvimento típico podem revelar características não esperadas para o desenvolvimento do processamento sensorial. Justificando a necessidade do acompanhamento dos mesmos com o intuito de prevenir e identificar precocemente possíveis atrasos no desenvolvimento ${ }^{14,15}$.

As condições de saúde do bebê é um fator intrínseco que interfere no desenvolvimento, porém o ambiente, fator extrínseco, pode influenciar no desenvolvimento sensorial do bebê. A relação entre bebê e ambiente é uma combinação que pode influenciar de forma positiva e/ou negativa no desenvolvimento do processamento sensorial ${ }^{14}$. Para mensurar a maneira como o bebê interage com o ambiente e responde ao mesmo por meio de comportamentos Dunn ${ }^{4}$, indica a avaliação dos quadrantes do processamento sensorial, que equivalem à harmonia entre o limiar neurológico e interação dos fatores ambientais e sensoriais do bebê.

Os resultados deste estudo indicaram que no quadrante "Exploração", que corresponde a como o bebê interage com a intensidade dos estímulos durante a atividade, alguns bebês avaliados apresentaram tanto uma hiporresponsividade (30\%), quanto uma hiperresponsividade (15\%). A "Esquiva" é representada por como o bebê reage a novos estímulos e situações desconhecidas, na qual $27 \%$ apresentaram um limiar abaixo do esperado para idade, e no quadrante "Sensibilidade", que avalia as reações frente aos estímulos, $12 \%$ dos bebês avaliados apresentaram um limiar abaixo do esperado ${ }^{4,14}$. Como os participantes são bebês com o desenvolvimento típico, tais resultados nos indicam uma alteração no processamento sensorial e/ou no ambiente em que ele está inserido.

A dinâmica dos fatores intrínsecos e extrínsecos para o desenvolvimento do bebê é um processo contínuo. É no ambiente familiar que o bebê recebe os primeiros estímulos através dos cuidados parentais e ofertas de brinquedos e situações que favorecerão a sua evolução. Sendo esta dinâmica a base para o desenvolvimento do bebê ${ }^{16}$. 
O estudo de Almeida et al. ${ }^{7}$ comparou as relações entre o desempenho motor e as características do ambiente familiar (espaço físico, atividades diárias, brinquedos) de lactentes residentes em duas regiões do Brasil e destacou a necessidade de avaliar os aspectos ambientais de famílias de bebês para orientar a prática clínica, seja no aspecto preventivo, educativo ou de reabilitação.

O Araújo et al. ${ }^{17}$ objetivou caracterizar o DNPM de crianças com até três anos participantes do Programa Leite das Crianças e relacionar com aspectos individuais, familiares, socioeconômicos e de estimulação da criança nos diferentes ambientes. Utilizaram a AHMED com 19 crianças de 7 a 35 meses e obteve como resultado 18,20\% como "menor do que adequada" as oportunidades ambientais que favoreçam o desenvolvimento motor do bebê.

Silva et al. ${ }^{18}$ propuseram a análise da relação entre as oportunidades de estimulação motora no ambiente familiar e o desenvolvimento motor de crianças. Obtiveram resultados similares a este estudo que classificaram as residências dos bebês como $67 \%$ "média" e $14 \%$ como "fraca" as oportunidades ambientais oferecidas para o bebê. Avaliar o ambiente doméstico é de grande valia para direcionar possíveis orientações e intervenções, a fim de favorecer o desenvolvimento do bebê ${ }^{6}$. Mesmo que Araújo et al. ${ }^{17}$ obtiveram um percentual de classificação inferior a estes estudos, cabe salientar que todo resultado que possa interferir no desenvolvimento do bebê, convêm um monitoramento.

Neste estudo, a categoria "Espaço externo", identificou que as oportunidades das residências foram classificadas como $19 \%$ sendo "fraca" e $38 \%$ "muito fraca". Tal categoria correspondente às características arquitetônicas do ambiente, como tipos de superfícies e inclinações e escadas, para que o espaço externo existente seja adequado para o bebê seriam necessárias despesas com reformas e reestruturação das residências. Este fato evidencia a necessidade de áreas públicas de lazer que ofereçam um espaço externo para minimizar os efeitos das baixas oportunidades de desenvolvimento no ambiente externo das residências ${ }^{9,19}$.

Nas avaliações do "espaço interno" mais da metade obtiveram resultados entre "muito fraca" $(6 \%)$, "fraca" $(21 \%)$ e "média" (27\%) as oportunidades ambientais oferecidas pelo ambiente interno das residências. Araújo et al. ${ }^{17}$ uma residência foi classificada como "muito fraca". O ambiente interno é o primeiro espaço de interação do bebê, este permite um bom desenvolvimento para bebê desde cedo. A qualidade do ambiente oferecido pelo espaço físico (espaço interno e espaço externo) não corresponde à percepção das famílias de bebês, tendo a visão de que sua residência oferece estímulos suficientes para seu filho?.

Pereira9 afirmou que as "variedades de estímulos" serem caracterizadas como "muito boa" nos indica uma conscientização dos pais sobre a importância do bebê ter contato com diferentes tipos de estímulos que permitem a exploração de objetos, livros e brinquedos, assim, proporcionando uma gama de oportunidades que favoreçam o desenvolvimento. Porém em alguns casos não é visível essa conscientização, pois os resultados indicam uma quantidade de residências classificadas como "muito fraca" $(5 \%)$, "fraca" (14\%) e "média" (3\%) a variedade de estímulos oferecidos ao bebê.

Os resultados obtidos das categorias "brinquedos para motricidade fina" e "brinquedos para motricidade grossa" foram negativos, ambas as categorias tiveram como classificação majoritária "fraca" e "muito fraca", evidenciando um déficit importante nessas dimensões, resultados semelhantes ao estudo de Araújo et al. ${ }^{17}$ que obteve a classificação de "muito fraca" e de Pereira ${ }^{9}$ com os resultados de "fraca" os brinquedos para estimular a motricidade fina e grossa dos bebês.

Os brinquedos de motricidade fina e grossa são essenciais para manutenção e estimulação do desenvolvimento da criança, "a motricidade fina abrange movimentos manuais com visão (coordenação óculo-manual) e a precisão de pequenos grupos musculares (músculos das mãos e dos dedos)". A motricidade fina e grossa são habilidades essenciais para que as crianças brinquem e explorem diferentes objetos através da estimulação, e isso engloba tipos de materiais, oferecida por parte das famílias, com o objetivo de garantir um bom desenvolvimento das habilidades motoras ${ }^{9}$.

\section{CONCLUSÕES}

Conclui-se a necessidade do monitoramento do desenvolvimento de bebês sem indicativos de atrasos no desenvolvimento, pela Atenção Primária à Saúde. Vista que existem áreas do processamento sensorial nas quais os bebês apresentam alterações no seu desenvolvimento, menor e/ou maior do que o esperado para idade, podendo interferir nas atividades diárias do bebê. Aplica-se também sobre a avaliação das oportunidades ambientais presentes nas residências dos bebês. Foram evidentes os déficits no ambiente, principalmente no espaço físico e nos brinquedos que estimulam a motricidade fina e grossa. Esse acompanhamento tem o caráter educativo e preventivo sobre possíveis atrasos no desenvolvimento infantil.

Agradecimento: À Pró-Reitoria de Extensão Universitária da Universidade Estadual Paulista (PROEX - UNESP) e ao Programa Institucional de Bolsas de Iniciação Científica (PIBIC) por todo apoio e incentivo concedido por meio de bolsas e fomento. 
Britto LB, et al. Processamento sensorial e oportunidades para o desenvolvimento de bebês. Rev Ter Ocup Univ São Paulo. 2020 jan.-dez.;31(1-3):9-16.

Contribuição das Autoras: Todos as autoras foram responsáveis pela concepção, redação e revisão do texto, organização de fontes e análises. Todos as autoras aprovaram a versão final do texto.

\section{REFERÊNCIAS}

1. Fisher AC, Murray E, Bundy A. Sensory integration: theory and practice. Philadelphia: FA Davis Company; 1991.

2. Serrano PA. Integração sensorial: No desenvolvimento e aprendizagem da criança. Lisboa: Papa- Letras; 2016.

3. Machado ACCP, Oliveira SR, Magalhães LC, Miranda DM, Bouzada MCF. Processamento sensorial no período da infância em crianças nascidas pré-termo: revisão sistemática. Rev Paul Pediatr. 2017;35(1):92-101. https://doi.org/10.1590/1984$0462 / ; 2017 ; 35 ; 1 ; 00008$

4. Dunn W. Perfil sensorial 2: manual do usuário. São Paulo: Pearson Clinical Brasil; 2017.

5. Pedrosa C, Caçola P, Carvalhal MIMM. Fatores preditores do perfil sensorial de lactentes dos 4 aos 18 meses. Rev Paul Pediatr. 2015; 33(2):160-6. https://doi.org/10.1016/j.rpped.2014.11.016

6. Caçola PM, Gabbard C, Montebelo MIL, Santos DCC. The new affordances in the home environment for motor development infant scale (AHEMD-IS): versions in English and Portuguese languages. Braz J Phys Ther. 2015;19(6):507-27. https://doi.org/10.1590/bjpt-rbf.2014.0112

7. Almeida TGA, Caçola PM, Gabbard C, Correr MT, Valela GB Jr, Santos DCC. Comparações entre o desempenho motor e oportunidades de estimulação motora no ambiente domiciliar de lactentes residentes nas regiões Sudeste e Norte do Brasil. Fisioter Pesqui. 2015;22(2):142-7. https://doi.org/10.590/ 1809-2950/13306322022015.

8. Caçola P, Gabbard C, Santos DC, Batistela AC. Development of the affordances in the home environment for motor development-infant scale. Pediatr Int. 2011;53(6):820-5. https://doi.org/10.1590/bjpt-rbf.2014.0112

9. Pereira, LGD. Oportunidades de estimulação motora do ambiente familiar: um estudo de caso com crianças dos 18 aos 42 meses [tese]. Faro, Portugal: Universidade do Algarve; 2018.

10. Molini-Avejonas DR, Rodon-Melo S, Batista ER, Souza AC, Dias DC, Samelli AG. Atenção básica como ordenadora do cuidado ao bebê de risco para alterações do neurodesenvolvimento. CoDAS. 2018;30(3):00302. http://dx.doi.org/10.1590/2317-1782/20182017064.

11. Oliveira LL, Costa VMR, Requeijo MR, Rebolledo RS, Pimenta AF, Lemos SMA. Desenvolvimento infantil:

Recebido em: 30.06 .2020

Aceito em: 21.09.2020 concordância entre a caderneta de saúde da criança e o manual para vigilância do desenvolvimento infantil. Rev Paul Pediatr. 2012;30(4):479-85. http://dx.doi.org/10.1590/ S0103-05822012000400004

12. Freitas M, Kernkraut AM, Guerreiro SMA, Akopian STG, Murakami SH, Madaschi V, et al. Acompanhamento de crianças prematuras com alto risco para alterações do crescimento e desenvolvimento: uma abordagem multiprofissional. Einstein, 2010;8(2):180-6. https://doi. org/10.1590/s1679-45082010ao1569

13. Almohalha L. Tradução, adaptação cultural e validação do Infant Sensory 2 e do ToddlerSensory Profile 2 para crianças brasileiras de 0 a 35 meses [tese]. Ribeirão Preto (SP): Escola de Enfermagem de Ribeirão Preto/USP; 2018.

14. Cabral TI, Silva LGP, Tudella E, Martinez CMS. Motor development and sensory processing: A comparative study between preterm and term infant. Res Develop Disabil. 2015;36:102-7. https://doi.org/10.1016/j.ridd.2014.09.018

15. Beltrame VH, Moraes AB, Souza APR. Perfil sensorial e sua relação com risco psíquico, prematuridade e desenvolvimento motor e de linguagem por bebês de 12 meses. Rev Ter Ocup Univ São Paulo. 2018; 29(1):8-18. https://doi.org/10.11606/ issn.2238-6149.v29i1p8-18

16. Morais RLS, Carvalho AM, Magalhães LC. A influência do contexto ambiental no desenvolvimento de crianças na primeira infância: estudos brasileiros. J Phys Educ. 2016;27:e2714

17. Araújo LB, Novaloski KRM, Bastos MSC, Mélo TR, Israel VL. Caracterização do desenvolvimento neuropsicomotor de crianças até três anos: o modelo da CIF no contexto do NASF1. Cad Bras Ter Ocup. 2018; 26(3):538-57. https://doi. org/10.4322/2526-8910.ctoAO1183

18. Silva WR, Lisboa T, Ferrari EP, Freitas KTD, Cardoso FL. Opportunities for motor stimulation in the home environment of children. J Hum Growth Dev. 2017;27(1):84-90. http://dx.doi.org/10.7322/jhgd.127659

19. Soares ES, Flores FS, Katzer JI, Valentini NC, Corazza ST, Copetti F. Análise das oportunidades de estimulação motora em ambientes domiciliares na região central do Rio Grande do Sul. Rev Bras Educ Fís Esporte. 2015;29(2):279-88. https://doi.org/10.1590/1807-55092015000200279 